\title{
Importance of tuberin in carcinogenesis (Review)
}

\author{
MINGWEI JIN, QI AN and LEI WANG \\ Department of Pediatric Internal Medicine, Xuzhou Children's Hospital, Xuzhou, Jiangsu 221002, P.R. China
}

Received March 21, 2017; Accepted June 26, 2017

DOI: $10.3892 / 01.2017 .6490$

\begin{abstract}
The cell cycle is a dynamic process with multiple phases regulating cell growth. The proper regulation is essential for avoiding errors and activation of cell death. Tumour suppressor proteins, including tuberin, are crucial in coordinating adequate cell growth and properly timed cell division. So, the present review article is focused on the latest aspects of the tuberin in the process of carcinogenesis. The PubMed was the main database used for the collection of latest data relating to multiple aspects of tuberin especially in context of cancer. Most of the recent studies revealed that mutation, truncation, and deregulation of the tuberin protein could definitely lead to cancer. Recent studies are also devoted to explore implications towards better understanding the progression of disease involving mis-regulated tuberin.
\end{abstract}

\section{Contents}

1. Introduction

2. Tuberin in G1/S transition

3. Nutrient and growth factor sensing proteins

4. Tuberin regulates downstream cell growth proteins

5. Tuberin and CCNB1 regulate mitotic onset

6. Tuberin is implicated in tumour formation

7. Treatment options

8. Conclusions

\section{Introduction}

The cell cycle is a tightly regulated process leading to proper cell growth and division (1). The cell cycle is a vigorous process and many cellular proteins, markedly the cyclins and their cyclin-dependent kinase (CDK) partners, tightly regulate the process (2). CDKs fall into a family of serine/threonine

Correspondence to: Dr Qi An, Department of Pediatric Internal Medicine, Xuzhou Children's Hospital, 18 Sudibei Road, Xuzhou, Jiangsu 221002, P.R. China

E-mail: aiqiangel@yeah.net

Key words: tuberin, cancer, cell cycle protein kinases, whose activity ultimately leads to cell cycle advancement. CDKs are expressed at relatively constant levels throughout the cell cycle and are regulated largely through post-translational modifications. The kinase activity of a CDK is dependent upon cyclin binding. In the absence of cyclin binding, a CDK is inactive due to blockage of the CDK active site by the T-loop. CDKs are also regulated by phosphorylation and dephosphorylation on conserved tyrosine (Y) and threonine (T) residues. Phosphorylation on T14 and Y15 by Myt1 and Weel kinases, respectively, render a CDK inactive.

Phosphorylation on these residues ultimately inhibits adenosine 5'-triphosphate (ATP) binding and full CDK activation (3). Phosphatase activity by Cdc25 on these two sites is essential for CDK1 activation and cell cycle progression overall. Following cyclin binding, CDK-activating enzymes phosphorylate a conserved threonine residue within the T-loop. Phosphorylation by CAK induces a conformational change in the CDK resulting in enhanced cyclin binding and complete activation. Upon complete activation, CDKs are able to phosphorylate specific protein substrates. Similarly, cyclins are a family of temporally regulated cell cycle proteins that can be categorized by type (4). Cyclin proteins are able to interact with CDKs through a conserved protein motif, known as the cyclin box. Classically, there are D-, E-, A- and B-type cyclins. Unlike their CDK partners, many cyclin proteins are expressed in a cyclical fashion, with levels rising and falling through progression of the cell cycle (5). At the onset of G1, levels of D-type cyclins rise and are able to bind CDK4 and CDK6. Co-currently, through S-phase levels of cyclin A rise and association with CDK2 is observed into G2. Subsequently, in late $\mathrm{G} 2$, the cyclin A-CDK1 interaction promotes M-phase entry. Continued regulation and progression of mitosis is controlled by CDK1 interactions with cyclin B1 (CCNB1) and B2 (6). Cyclin proteins, with the exception of D-type cyclins are heavily regulated first by their synthesis and second through ubiquitin-mediated degradation (7).

Cell cycle progression is also regulated spatially, via intracellular cyclin-CDK localization (8). CCNB1 forms a complex with CDK1 to control mitotic onset. The CCNB1, is located on chromosome $5 q 13.2$ and results in a $62 \mathrm{kDa}$ protein. The mammalian CCNB1 protein, contains the classical cyclin box domain (aa210-348), which allows for its direct CDK1 interaction. In 1998 Yang et al observed that during interphase, CCNB1 is actively shuttled from the nucleus to the cytoplasm until prophase begins. From here, CCNB1 protein accumulates in the nucleus only when the rate of CCNB1 import/export is altered (9). Surprisingly, CCNB1 lacks a classical nuclear 
localization signal, but contains a nuclear export signal (NES) within a region classically coined the cytoplasmic retention sequence (CRS). This CRS domain is of key importance since it contains five conserved serine residues, which are phosphorylation targets. It was originally thought that these sites, when phosphorylated, facilitate nuclear accumulation (10). CRM1 mediated export is an extremely important regulator of CCNB1 localization. Shuttling of CCNB1 from the nucleus to the cytoplasm is in a constant equilibrium (10).

The aforementioned NES is located within the CRS region (aa88-154) of CCNB1 (8). The CCNB1-CDK1 complex is an extremely important cell cycle activator at the G2/M transition because it is responsible for chromosome condensation, nuclear envelope breakdown, and mitotic spindle assembly (11). During S-phase, CCNB1 protein accumulation begins, peaking at prophase and metaphase, and significantly declining at the start of anaphase via degradation by the cyclosome/anaphase-promoting complex (APC) (12). However, hyper-phosphorylation of the mentioned serine residues results in hindering of NES activity and promotes nuclear accumulation. CCNB1 residues S126 and S128 are known CDK1 targets and are the first residues responsible for promoting B1 nuclear translocation. S147 is phosphorylated by polo-like kinase 1, a kinetochore localizing protein that is a known activator of the APC (13). These events; however, are not fully sufficient to prevent CCNB1 export, indicating additional mechanisms are involved in this complex process.

\section{Tuberin in G1/S transition}

Multiple proteins regulate cell cycle control and the tuberin is one of them. This large protein contains a variety of conserved domains responsible for its function reviewed by Rosner et al (14). Tuberin specifically contains two coiled-coil domains (aa346-371), a leucine zipper (aa75-107), a GTPase activating protein (GAP) homology domain (aa1517-1674) and a calmodulin domain (aa1740-1758). The most widely known role for tuberin is tumour suppression at the G1/S transition by responding to nutrient and growth factor stimuli. Tuberin in a complex with a protein hamartin form the tuberous sclerosis complex (TSC), which negatively regulates cell growth and proliferation (15). Tuberin, independent of hamartin, is known to interact with the important CKI, p27, which markedly inhibits the G1/S transition. Tuberin accomplishes this action by binding to the CDK2 inhibitor, p27, protecting it from ubiquitin-mediated degradation. Tuberin also allows for nuclear accumulation of p27 by interfering with the 14-3-3s-dependent CRS of p27. These results, taken together, demonstrate the ability of tuberin to negatively regulate the cell cycle at the G1/S transition (16). The hamartin is another protein that in association with tuberin forms a tumour suppressor complex. Hamartin has a large role in the stabilization of tuberin primarily by inhibiting its interaction with the ubiquitin ligase, HECT and RLD domain containing E3 ubiquitin protein ligase family member 1, HERC1 (17).

\section{Nutrient and growth factor sensing proteins}

Together, tuberin and hamartin integrate levels of nutrients, growth factors, and energy stores to regulate cell growth and proliferation (18). This integration is important since it results in the inhibition of the activity of downstream tuberin targets (19). They accomplish this by responding to a variety of upstream proteins that regulate their protein-protein interactions as well as the phosphorylation, and subcellular localization of tuberin. Specifically, energy is sensed in the cell via ATP levels. In the event of high energy levels, the cell will be in an ATP high flux. Conversely, if energy levels fall low, an adenosine monophosphate (AMP) high flux will result. If high levels of AMP are present within the cell, AMP would bind to the AMP-activated protein kinase (AMPK) leading to cell growth inhibition (20).

Mitogen stimulation in the form of growth factors, cytokines, or hormones is also communicated through tuberin and the TSC. Growth factor levels of insulin and insulin-like growth factor (IGF) are transmitted through IGF transmembrane receptors binding. Transmembrane activation results in insulin receptor substrate recruitment and subsequent phosphoinositide 3-kinase (PI3K) activation as reviewed (18). PI3K exerts its affects through lipids, specifically through phosphorylation of membrane-associated phosphatidylinostitol 3-phosphate (PtdIns 3). These events recruit phosphoinositidedependent kinase-1 to active membrane associated lipids. Once bound, PDK1 becomes active and is allowed to activate protein kinase B (Akt) (21). The serine threonine kinase, Akt, has the ability to bind and negatively regulate the tuberin protein. Inhibitory phosphorylation by Akt occurs on S939 and T1462 of the tuberin protein in the event of high nutrient and insulin signaling (22).

Conversely, cell growth regulation by tuberin is not only controlled through Akt, but also through mitogen activated protein kinase/extracellular signaling related kinase (Erk) signaling (23). Epidermal growth factor is known to bind the transmembrane-associated epidermal growth factor receptor This binding induces intrinsic membrane activation, through tyrosine phosphorylation, resulting in signal transduction events involving the GTPase, and Ras. Dual phosphorylation on tuberin through Erk decreases the affinity of tuberin for hamartin, resulting in enhanced S6K activity through mTOR and strikingly, tuberin modification via Erk has significantly increased effects on cell proliferation and oncogenic transformation (24).

\section{Tuberin regulates downstream cell growth proteins}

Tuberin is a fundamental player in the regulation of cell growth and protein synthesis through its downstream inhibition of mTOR during the G1/S transition (15). Tuberin contains a C-terminal GAP domain allowing its GTPase activating functions. Tuberin has the ability to bind Ras homolog enriched in brain (Rheb). Rheb, a member of the Ras super family, functions as small GTPase protein, which shuttles between its active (GTP bound) and inactive (GDP bound) form. GTPases can weakly and slowly hydrolyze their bound GTP to GDP. Tuberin acts as a GAP towards Rheb through direct binding. The GAP function of tuberin accelerates the G-proteins built in hydrolysis timer. Specifically, tuberin binds the G-protein, Rheb, directing a conformational change, which allows for nucleophilic attack and quick hydrolysis, rendering Rheb in the inactive GDP form. GDP bound Rheb is unable to bind and activate mTOR, thereby halting an increase in cell growth. In 
this manner, tuberin GAP activity is prevented by Akt phosphorylation on sites previously mentioned (25).

When active, Rheb acts as a mediator, inhibiting mTOR repression, by abrogating the association of MTOR with the endogenous inhibitor FKBP38 (26). The mTOR protein is a complex protein involved in many more cellular events than described here. Briefly, mTOR retains hierarchical control over protein synthesis, cell growth and autophagy, by responding to upstream nutrient, growth factor and amino acid stimuli (27). mTOR is a large serine/threonine protein kinase that is composed of two distinct complexes: mTORC1 and mTORC2 (28). Both complexes are known to localize to distinct subcellular components, contributing to their differential roles within the cell (29). The mTORC2 subunit, with its rapamycin insensitive partner, rictor, is known to regulate the actin cytoskeleton and provide input to cellular metabolism through feedback to Akt (30). The more described subunit, mTORC1, accomplishes the activation of protein synthesis by upstream regulation of ribosomal protein S6 kinase $\beta-1$ (p70S6K) and 4E binding protein 1 (4E-BP1) (28). mTORC1 phosphorylates $\mathrm{S} 6 \mathrm{~K}$ resulting in activation of the ribosomal S6 protein subunit leading to increase translation and cellular growth (31). Concomitantly, mTORC1 phosphorylates and inhibits 4E-BP1, thereby allowing the eukaryotic initiation factor $4 \mathrm{E}$ (eIF4E) to be free, participate in mRNA stabilization by cap binding and initiate 5 ' cap-dependent translation. These events also lead to an increase in protein synthesis, cell size, and cell cycle advancement (32).

mTOR involvement in cell growth is heavily dependent on available amino acids and growth factor levels; however, when the cell is found in a nutrient poor state, the initiation of events such as autophagy may occur. Autophagy is described as a process in which cells undergo rearrangement and sequester a portion of cytoplasm, organelles and intracellular proteins for lysosomal degradation in order to maintain cellular homeostasis and to produce energy in times of stress (33). Classically, active mTOR negatively regulates the onset of autophagy through mammalian autophagy kinase [uncoordinated (Unc) 51-like kinase 1/2 (ULK1/2)] inhibitory phosphorylation. Inhibitory release of ULK allows downstream activation of autophagy-related gene (Atg)13, FIP200, and ULK itself; ultimately resulting in a cascade of Atg activation and biosynthesis of the autophagosome (34). Autophagy can be initiated by AMPK indirectly through tuberin phosphorylation and mTORC1-mediated signals (35). Moreover, autophagy is activated downstream and independently of tuberin, by AMPK-dependent raptor phosphorylation. It has been found recently that the autophagy substrate p62/sequestosome 1 are critical components of TSC/mTORC1 driven tumour development (33). In particular, the survival of TSC2-null cells is dependent upon autophagy in an mTORC1-dependent fashion. Nonetheless, it is clear that mTORC1 is a convergent mechanism through which autophagy is regulated. It is noteworthy to think about the possibilities that tuberin and the TSC may have on autophagy initiation independently of mTOR.

\section{Tuberin and CCNB1 regulate mitotic onset}

In addition to inhibitory roles with cell cycle proteins and growth inhibition at the G1/S transition, our laboratory has elucidated a novel and imperative role for tuberin at the G2/M transition. Since mitosis is directly responsible for cell division, cells must grow to double their original size and have appropriately segregated DNA. The exact mechanisms regulating the transition of cell growth to cell division are not fully understood.

In 2011, Fidalgo da Silva et al (36) observed, through a yeast 2 hybrid screen, that tuberin forms a transient complex with CCNB1at the $\mathrm{G} 2 / \mathrm{M}$ transition, providing an intriguing mechanism through which CCNB1 localization is regulated. Using small fragments of tuberin (aa600-746), positive interactions were seen with the CRS region of CCNB1 protein. Fishing with a small fragment of CCNB1 containing the CRS region yielded a small fragment of tuberin spanning from amino acids 610-746. It is now established that this region of tuberin is responsible for both CCNB1 binding and cytoplasmic localization of CCNB1. The data present the possibility that tuberin can regulate premature mitotic onset. The tuberin-CCNB1 complex is known to associate with both hamartin and CDK1, presenting the possibility that feedback can be provided to regulate G1/S. Utilizing CCNB1 NES variants, it was seen that nearly $100 \%$ of the cyclin variant, CCNB15E (which mimics fully phosphorylated CCNB1), localizes to the nucleus after $24 \mathrm{~h}$.

\section{Tuberin is implicated in tumour formation}

Tuberous sclerosis (TSC) is known to arise from mutations in both the TSC1 and TSC2 genes; however, mutations in TSC2 result in more severe disease related phenotypes compared to that of TSC1 (37). TS is characterized by the formation of benign tumours, referred to as hamartomas, that occur in a variety of organ types such as the brain, heart, skin, kidney and lung. TS most frequently occurs during development and displays itself in early childhood. Traditionally, TS is considered an autosomal-dominate disorder acquired through inheritance; however, approximately two-thirds of TS cases arise from sporadic germ-line mutations. Disease prevalence affects approximately 1 in 6,000 live births annually and is estimated to affect roughly 1.5 million individuals worldwide (38). The tuberin protein has proven itself a fundamental and highly important protein. Deletion, truncation, or mutation can result in very severe cellular outcomes. This protein is known to be essential for neural tube closure and TSC2 gene deletion results in embryonic lethality at day 10.5 in Eker rat models (39).

The severity and prognosis of the disease is widely variable (40). Over 95\% of patients have dermalogical manifestations, such as facial angiofibroma, which often causes no detriment to life (41). However, manifestations affecting the brain, such as cortical tubers, subependymal nodules or subependymal giant cell astrocytomas can cause blood vessel obstruction and cerebrospinal fluid accumulation, which can be lethal (42). The central nervous system is affected in nearly $50 \%$ of TS sufferers and these mentioned brain abnormalities can result in forms of autism, epilepsy (in 60-90\% of cases), mild to severe learning difficulties and behavioural disorders, such as attention deficit hyperactive disorder (43). Conversely, TS outcomes such as angiomyolipoma developing within the kidney may lead to kidney failure and impaired function, haemorrhaging and possible development of 
carcinomas. In addition to the role of tuberin in the benign tumours of TS patients, tuberin mutations have also been found to cooperate with oncogenic mutations aiding in the initiation and progression of a number of malignant cancers including medulloblastoma renal cell carcinomas and lung, kidney, and breast cancers (44-46). The tremendous variability in the severity of the tumour formed in TS are ascribed to the fact that mutations in TSC2 are known to span the entire length of the gene, resulting in a plethora of possible tuberin protein mutations. It is notable however that mutational hotspots have been identified, and that one of these is located within the CCNB1 binding domain of tuberin (36).

Specifically, tuberin functions as a chaperone protein by maintaining the complex in the soluble form and by preventing the self-aggregation of hamartin. In 2005, Nellist et al (47) found that phosphorylation of known Akt target sites was inhibited in the mentioned tuberin mutants. Data here suggest that clinically relevant tuberin mutations likely render the tuberin protein in conformations that are unfavourable for important regulatory modifications, indicative of impaired tuberin function. In addition, mutations such as these have been associated with increased S6K phosphorylation and increased Rheb-GTPase activity compared to that of wildtype tuberin. However, mutations residing outside the CCNB1 binding domain, do not elicit the same effects and disease severity. Moreover, immunofluorescence studies and protein binding densitometry analysis confirm that C696Y significantly exhibits more than a $50 \%$ decrease in binding affinity to CCNB1 compared to that of wild-type tuberin (36). This finding may indicate increased cell cycle progression and may explain the increase in nuclear localization of CCNB1. In a recent medical study, Yu et al (48) performed mutational analysis on a family affected with TS. Sequencing analysis revealed a novel tuberin truncation mutant that arises from a deletion at exon 24 in the TSC2 gene. This mutation results in a 946 amino acid protein, which is expected to have intact hamartin binding. A mutation in the TSC2 gene such as this lacks the C-terminal GAP domain, transcriptional activation domains, and many critical phosphorylation sites, including the important regulatory Erk and Akt phosphorylation sites. Naturally, it could be expected that loss of these critical domains can impair proper tuberin function and lead to uncontrolled growth resulting in tumour initiation.

\section{Treatment options}

Unfortunately, TS patients have a minimal number of accessible treatment options. The growth of these benign tumours must be monitored closely and if possible, surgically removed. One of the most prevalent treatment options for life-threatening manifestations is treatment with mTOR inhibitors such as rapamycin, sirolimus and everolimus (49). Many clinical trials are underway to increase efficacy of these treatments and determine additional mTOR inhibitors. As explained, there is a vast amount of research in this field targeted towards understanding the tuberin role with respect to protein synthesis and regulation of the G1/S transition, but it is imperative that we better understand the cell division regulation of tuberin at the G2/M transition, as traditional measures of treating TS may not be applicable to this transient interaction.

\section{Conclusions}

The involvement of tuberin at the onset of mitosis is still poorly understood. Given the diversity in the phenotypes found with different mutations, future research should focus on mechanistic studies on multiple severe mutations found within tuberin.

\section{References}

1. Cannell IG, Merrick KA, Morandell S, Zhu CQ, Braun CJ, Grant RA, Cameron ER, Tsao MS, Hemann MT and Yaffe MB: A pleiotropic RNA-binding protein controls distinct cell cycle checkpoints to drive resistance of p53-defective tumors to chemotherapy. Cancer Cell 28: 623-637, 2015.

2. Badjatia N, Park SH, Ambrósio DL, Kirkham JK and Günzl A: Cyclin-dependent kinase CRK9, required for spliced leader trans splicing of pre-mRNA in trypanosomes, functions in a complex with a new L-type cyclin and a kinetoplastid-specific protein. PLoS Pathog 12: e1005498, 2016.

3. Gaggioli V, Zeiser E, Rivers D, Bradshaw CR, Ahringer J and Zegerman P: CDK phosphorylation of SLD-2 is required for replication initiation and germline development in C.elegans. $\mathrm{J}$ Cell Biol 204: 507-522, 2014.

4. Vermeulen K, Van Bockstaele DR and Berneman ZN: The cell cycle: A review of regulation, deregulation and therapeutic targets in cancer. Cell Prolif 36: 131-149, 2003.

5. Nurse PM: Nobel lecture. Cyclin dependent kinases and cell cycle control. Biosci Rep 22: 487-499, 2002.

6. Arellano M and Moreno S: Regulation of CDK/cyclin complexes during the cell cycle. Int J Biochem Cell Biol 29: 559-573, 1997.

7. Kontopidis G, Wu SY, Zheleva DI, Taylor P, McInnes C, Lane DP, Fischer PM and Walkinshaw MD: Structural and biochemical studies of human proliferating cell nuclear antigen complexes provide a rationale for cyclin association and inhibitor design. Proc Natl Acad Sci USA 102: 1871-1876, 2005.

8. Porter LA and Donoghue DJ: Cyclin B1 and CDK1: Nuclear localization and upstream regulators. Prog Cell Cycle Res 5: 335-347, 2003.

9. Yang J, Bardes ES, Moore JD, Brennan J, Powers MA and Kornbluth S: Control of cyclin B1 localization through regulated binding of the nuclear export factor CRM1. Genes Dev 12: 2131-2143, 1998.

10. Hagting A, Jackman M, Simpson K and Pines J: Translocation of cyclin B1 to the nucleus at prophase requires a phosphorylation-dependent nuclear import signal. Curr Biol 9: 680-689, 1999.

11. Ciemerych MA and Sicinski P: Cell cycle in mouse development. Oncogene 24: 2877-2898, 2005.

12. Chang DC, Xu N and Luo KQ: Degradation of cyclin B is required for the onset of anaphase in Mammalian cells. J Biol Chem 278: 37865-37873, 2003.

13. Toyoshima-Morimoto F, Taniguchi E, Shinya N, Iwamatsu A and Nishida E: Polo-like kinase 1 phosphorylates cyclin B1 and targets it to the nucleus during prophase. Nature 410: 215-220, 2001.

14. Rosner M, Hanneder M, Siegel N, Valli A and Hengstschläger M: The tuberous sclerosis gene products hamartin and tuberin are multifunctional proteins with a wide spectrum of interacting partners. Mutat Res 658: 234-246, 2008.

15. Tee AR, Manning BD, Roux PP, Cantley LC and Blenis J: Tuberous sclerosis complex gene products, Tuberin and Hamartin, control mTOR signaling by acting as a GTPase-activating protein complex toward Rheb. Curr Biol 13: 1259-1268, 2003.

16. Rosner M, Freilinger A, Hanneder M, Fujita N, Lubec G, Tsuruo $\mathrm{T}$ and Hengstschläger M: p27Kip1 localization depends on the tumor suppressor protein tuberin. Hum Mol Genet 16: 1541-1556, 2007a.

17. Chong-Kopera H, Inoki K, Li Y, Zhu T, Garcia-Gonzalo FR, Rosa JL and Guan KL: TSC1 stabilizes TSC2 by inhibiting the interaction between TSC 2 and the HERC1 ubiquitin ligase. J Biol Chem 281: 8313-8316, 2006.

18. Orlova KA and Crino PB: The tuberous sclerosis complex. Ann N Y Acad Sci 1184: 87-105, 2010.

19. Inoki K, Zhu T and Guan KL: TSC2 mediates cellular energy response to control cell growth and survival. Cell 115: 577-590, 2003. 
20. Shaw RJ: LKB1 and AMP-activated protein kinase control of mTOR signalling and growth. Acta Physiol (Oxf) 196: 65-80, 2009.

21. Manning BD and Cantley LC: United at last: The tuberous sclerosis complex gene products connect the phosphoinositide 3-kinase/Akt pathway to mammalian target of rapamycin (mTOR) signalling. Biochem Soc Trans 31: 573-578, 2003.

22. Rosner M, Freilinger A and Hengstschlager M: Akt regulates nuclear/cytoplasmic localization of tuberin. Oncogene 26 : 521-531, 2007.

23. Ma L, Chen Z, Erdjument-Bromage H, Tempst P and Pandolfi PP: Phosphorylation and functional inactivation of TSC2 by Erk implications for tuberous sclerosis and cancer pathogenesis. Cell 121: 179-193, 2005.

24. Ma L, Teruya-Feldstein J, Bonner P, Bernardi R, Franz DN, Witte D, Cordon-Cardo C and Pandolfi PP: Identification of S664 TSC2 phosphorylation as a marker for extracellular signal-regulated kinase mediated mTOR activation in tuberous sclerosis and human cancer. Cancer Res 67: 7106-7112, 2007.

25. Li Y, Inoki K, Vikis H and Guan KL: Measurements of TSC2 GAP activity toward Rheb. Methods Enzymol 407: 46-54, 2006.

26. Bai X, Ma D, Liu A, Shen X, Wang QJ, Liu Y and Jiang Y: Rheb activates mTOR by antagonizing its endogenous inhibitor, FKBP38. Science 318: 977-980, 2007.

27. Beugnet A, Tee AR, Taylor PM and Proud CG: Regulation of targets of mTOR (mammalian target of rapamycin) signalling by intracellular amino acid availability. Biochem J 372: 555-566, 2003.

28. Wullschleger S, Loewith R and Hall MN: TOR signaling in growth and metabolism. Cell 124: 471-484, 2006.

29. Betz C and Hall MN: Where is mTOR and what is it doing there? J Cell Biol 203: 563-574, 2013.

30. Sarbassov DD, Ali SM, Kim DH, Guertin DA, Latek RR, Erdjument-Bromage H, Tempst P and Sabatini DM: Rictor, a novel binding partner of mTOR, defines a rapamycin-insensitive and raptor-independent pathway that regulates the cytoskeleton. Curr Biol 14: 1296-1302, 2004.

31. Garami A, Zwartkruis FJ, Nobukuni T, Joaquin M, Roccio M, Stocker H, Kozma SC, Hafen E, Bos JL and Thomas G: Insulin activation of Rheb, a mediator of mTOR/S6K/4E-BP signaling, is inhibited by TSC1 and 2. Mol Cell 11: 1457-1466, 2003.

32. Dann SG, Selvaraj A and Thomas G: mTOR Complex1-S6K1 signaling: At the crossroads of obesity, diabetes and cancer. Trends Mol Med 13: 252-259, 2007.

33. Parkhitko A, Myachina F, Morrison TA, Hindi KM, Auricchio N Karbowniczek M, Wu JJ, Finkel T, Kwiatkowski DJ, Yu JJ, et al: Tumorigenesis in tuberous sclerosis complex is autophagy and p62/sequestosome 1 (SQSTM1)-dependent. Proc Natl Acad Sci USA 108: 12455-12460, 2011.

34. Klionsky DJ, Abeliovich H, Agostinis P, Agrawal DK, Aliev G, Askew DS, Baba M, Baehrecke EH, Bahr BA, Ballabio A, et al: Guidelines for the use and interpretation of assays for monitoring autophagy in higher eukaryotes. Autophagy 4: 151-175, 2008

35. Kim J, Kundu M, Viollet B and Guan KL: AMPK and mTOR regulate autophagy through direct phosphorylation of Ulk1. Nat Cell Biol 13: 132-141, 2011.
36. Fidalgo da Silva E, Ansari SB, Maimaiti J, Barnes EA, Kong-Beltran M, Donoghue DJ and Porter LA: The tumor suppressor tuberin regulates mitotic onset through the cellular localization of cyclin B1. Cell Cycle 10: 3129-3139, 2011.

37. Zeng LH, Rensing NR, Zhang B, Gutmann DH, Gambello MJ and Wong M: Tsc2 gene inactivation causes a more severe epilepsy phenotype than Tsc1 inactivation in a mouse model of tuberous sclerosis complex. Hum Mol Genet 20: 445-454, 2011.

38. Curatolo P, Bombardieri R and Jozwiak S: Tuberous sclerosis. Lancet 372: 657-668, 2008.

39. Rennebeck G, Kleymenova EV, Anderson R, Yeung RS, Artzt K and Walker CL: Loss of function of the tuberous sclerosis 2 tumor suppressor gene results in embryonic lethality characterized by disrupted neuroepithelial growth and development. Proc Nat Acad Sci USA 95: 15629-15634, 1998.

40. Wataya-Kaneda M, Tanaka M, Hamasaki T and Katayama I: Trends in the prevalence of tuberous sclerosis complex manifestations: An epidemiological study of 166 Japanese patients. PLoS One 8: e63910, 2013.

41. Kwiatkowski DJ, Palmer MR, Jozwiak S, Bissler J, Franz D, Segal S, Chen D and Sampson JR: Response to everolimus is seen in TSC-associated SEGAs and angiomyolipomas independent of mutation type and site in TSC1 and TSC2. Eur J Hum Genet 23: $1665-1672,2015$

42. Kohrman MH: Emerging treatments in the management of tuberous sclerosis complex. Pediatr Neurol 46: 267-275, 2012.

43. Ehninger D, de Vries PJ and Silva AJ: From mTOR to cognition Molecular and cellular mechanisms of cognitive impairments in tuberous sclerosis. J Intellect Disabil Res 53: 838-851, 2009.

44. Franz DN, Bissler JJ and McCormack FX: Tuberous sclerosis complex: Neurological, renal and pulmonary manifestations. Neuropediatrics 41: 199-208, 2010.

45. Bhatia B, Northcott PA, Hambardzumyan D, Govindarajan B, Brat DJ, Arbiser JL, Holland EC, Taylor MD and Kenney AM: Tuberous sclerosis complex suppression in cerebellar development and medulloblastoma: Separate regulation of mammalian target of rapamycin activity and p27 Kip1 localization. Cancer Res 69: 7224-7234, 2009.

46. Dabora SL, Jozwiak S, Franz DN, Roberts PS, Nieto A, Chung J, Choy YS, Reeve MP, Thiele E, Egelhoff JC, et al: Mutational analysis in a cohort of 224 tuberous sclerosis patients indicates increased severity of TSC2, compared with TSC1, disease in multiple organs. Am J Hum Genet 68: 64-80, 2001.

47. Nellist M, Burgers PC, van den Ouweland AM, Halley DJ and Luider TM: Phosphorylation and binding partner analysis of the TSC1-TSC2 complex. Biochem Biophys Res Commun 333: 818-826, 2005a.

48. Yu Z, Zhang X, Guo H and Bai Y: A novel TSC2 mutation in a Chinese family with tuberous sclerosis complex. J Genet 93: 169-172, 2014.

49. Kohrman MH: Emerging treatments in the management of tuberous sclerosis complex. Pediatr Neurol 46: 267-275, 2012. 